\title{
Qui a peur de la vague grise?
}

\section{Anna Sax}

Lic. oec. publ., MHA, membre de la rédaction

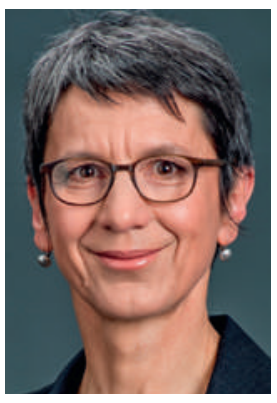

Je fais partie de la génération qui déferlera en «vague grise» sur notre pays d'ici 20 à 30 ans. Nous serons des centaines de milliers à errer atteints de démence, à assaillir les services d'urgence pour nos os brisés, à nous oublier dans nos sous-vêtements et à ralentir le trafic du fait de notre incapacité à monter et descendre des véhicules rapidement. Mais ce n'est pas tout: ma génération n'a pas mis d'enfants au monde et nous manquons donc de fils et de belles-filles pour prendre soin de nous durant nos vieux jours.

L'Office fédéral de la statistique estime que le nombre des plus de 80 ans doublera d'ici 2040. De telles prévisions et différentes interventions parlementaires ont amené le Conseil fédéral à diligenter un rapport sur l'avenir des soins de longue durée ${ }^{1}$, car il y a tout lieu de penser que les besoins en soins vont également exploser. Un colloque du think tank proche des organisations syndicales "Réseau de réflexion», début septembre, a fait de ce rapport le point de départ d'un débat sur les modèles de soins et de prise en charge. L'association faîtière des EMS, Curaviva, a entre autres exposé «Le modèle d'habitat \& de soins 2030», qui supprime en partie la dichotomie soins ambulatoires/stationnaires. Le modèle repose sur des centres de santé décentralisés, où des offres de services et de soins adaptées à des besoins changeants permettent de mener une vie autonome malgré la dépendance. De bons exemples pratiques en Suisse et à l'étranger ont été présentés. Il existe des approches prometteuses, des idées convaincantes. On peut par exemple citer «GrossmütterRevolution $»^{2}$, une plateforme pour les femmes politiquement engagées de la génération des grands-mères. Cela donne bon espoir.

Des soins de qualité ne sont bien sûr pas gratuits. Les coûts et le financement de l'aide et des soins à domicile ainsi que des établissements médico-sociaux continuent à soulever de vifs débats, même après l'introduction du nouveau financement des soins en 2011. Quelle doit être la part acquittée par les bénéficiaires des soins, par l'Etat, par la caisse-maladie? Faut-il une assu- rance des soins? Ces questions continueront de nous préoccuper, alors que les coûts ne sont probablement pas notre principal problème. Compte tenu de l'évolution démographique prévisible, ce serait une bonne idée de se demander qui, à l'avenir, sera encore en mesure de s'occuper des personnes âgées et dépendantes. Il faut d'une part des professionnels de la santé et d'autre part des filles, gendres, partenaires, neveux, amies et voisins prêts à donner un coup de main.

Et nous voilà de retour au renversement de la pyramide des âges. Il y a quelques années, l'Observatoire suisse de la santé (Obsan) a tracé une courbe frappante. Celle-ci montre le rapport entre le nombre de femmes de 35 à 60 ans et le nombre de personnes de plus de 80 ans, en partant du principe que les soins sont avant tout l'affaire des femmes. Aujourd'hui, pour chaque personne âgée, 3,5 filles ou belles-filles sont disponibles en tant que proches soignantes potentielles. En 2050, elles ne seront plus que 1,4. Même si la descendance masculine s'implique nettement plus qu'avant dans les soins, la génération du baby-boom va devoir trouver d'autres solutions pour ne pas passer ses vieux jours dans l'abandon et la solitude. Les seniors devront s'entraider, répartir leurs forces et recourir de manière ciblée aux services pour répondre aux besoins les plus urgents. Il leur faudra des amies et amis. Ils dépendront de concitoyennes et de concitoyens solidaires, prêts à dégager les moyens financiers nécessaires pour assurer des prestations professionnelles dans la vieillesse. Il faut espérer que les visions telles que le modèle d'habitat \& de soins 2030 de Curaviva se concrétiseront.

J'aurais de bonnes raisons d'avoir peur de vieillir, pourtant, ce n'est pas le cas. La vie peut prendre tellement de tournants inattendus, tant de choses ne se produisent pas comme prévu. Je verrai bien. Je ne sais même pas si je vivrai vraiment vieille. Il n'y a qu'une chose dont je suis sûre: je ne voudrai pas vivre dans une résidence partagée pour personnes âgées si je peux l'éviter. Je n'ai jamais été fan de colocation. 